\title{
Reflets
}

Revue ontaroise d'intervention sociale et communautaire

\section{Les femmes francophones dans le milieu ontarien de la violence faite aux femmes}

\section{Ghislaine Sirois}

Volume 4, numéro 1, printemps 1998

Intervention en contextes minoritaires

URI : https://id.erudit.org/iderudit/026202ar

DOI : https://doi.org/10.7202/026202ar

Aller au sommaire du numéro

Éditeur(s)

Reflets : Revue ontaroise d'intervention sociale et communautaire

ISSN

1203-4576 (imprimé)

1712-8498 (numérique)

Découvrir la revue

Citer cet article

Sirois, G. (1998). Les femmes francophones dans le milieu ontarien de la violence faite aux femmes. Reflets, 4(1), 151-163.

https://doi.org/10.7202/026202ar

Tous droits réservés (C) Reflets : Revue ontaroise d'intervention sociale et communautaire, 1998
Ce document est protégé par la loi sur le droit d'auteur. L'utilisation des services d'Érudit (y compris la reproduction) est assujettie à sa politique d'utilisation que vous pouvez consulter en ligne.

https://apropos.erudit.org/fr/usagers/politique-dutilisation/ 


\section{Les femmes francophones dans le milieu ontarien de la violence faite aux femmes ${ }^{1}$}

\section{Ghislaine Sirois}

Directrice, Action ontarienne contre la violence faite aux femmes

\section{Pourquoi ce texte}

Action ontarienne contre la violence faite aux femmes (AOcVF) est un regroupement provincial d'intervenantes et de survivantes d'expression française impliquées dans la lutte contre la violence faite aux femmes en Ontario. Au cours des années, nous avons obtenu une grande collaboration de la part des intervenantes et des développeur-e-s de services - dont plusieurs anglophones dans le domaine. Mais parfois, nous avons fait face à de l'incompréhension face à la réalité des femmes francophones, incompréhension manifestée par de la résistance, des «oublis», des préjugés et à l'occasion, par l'expression de mépris.

Nous voulons donc ici briser le silence qui entoure cette incompréhension et amener le débat dans les lieux de discussions des femmes de l'Ontario, tant pour les femmes francophones qui se sentent isolées et silencieuses par crainte de réactions négatives, que pour nos consoeurs dont la langue de communication en Ontario est l'anglais, afin qu'une compréhension accrue nous ouvre de part et d'autre les portes de collaborations nouvelles. 
Nous sommes d'avis que ces résistances, oublis, préjugés et expressions de mépris créent une forme d'oppression dirigée envers les femmes d'expression française en Ontario. Plusieurs pourraient réagir en pensant que les femmes francophones ne sont pas, à première vue, susceptibles d'appartenir à un groupe qui vit de l'oppression :

- parce que nous en connaissons toutes qui «ont réussi»;

- parce qu'un bon nombre jouissent de certains des privilèges alloués aux Nord-américaines de race blanche;

- parce qu'on oublie qu'il ne s'agit pas d'un groupe homogène, mais qu'on y trouve aussi des femmes de couleur, des femmes noires, âgées, jeunes, des femmes vivant avec un handicap, des lesbiennes, des femmes pauvres, de femmes appartenant à d'autres catégories qui ont fait historiquement l'objet de discrimination.

\section{Les pièges}

"L'oppression est construite à partir d'un enchevêtrement complexe aux manifestations diverses, certaines menaçant la survie l'acculturation des autochtones, le racisme, la violence contre les femmes par exemple d'autres menaçant la santé psychique et physique ou encore le sens d'appartenance.»
Une telle entreprise comporte des pièges, dont le premier serait pour les femmes francophones de se cantonner dans un rôle de victime. Au contraire, nous proposons à ces dernières d'identifier l'oppression dont elles sont victimes et d'utiliser les moyens mis à leur service.

Le second piège serait de vouloir établir une échelle du degré d'oppression. Ce serait là tomber dans une analyse simpliste des communautés, analyse que la bureaucratie renforce de peur d'une situation trop complexe à gérer. En effet, vers quel groupe de soutien devrait-elle orienter une femme francophone noire vivant avec un handicap et survivante d'agression sexuelle? L'oppression est construite à partir d'un enchevêtrement complexe aux manifestations diverses, certaines menaçant la survie — l'acculturation des autochtones, le racisme, la violence contre les femmes, par exemple - d'autres menaçant la santé psychique et physique ou encore le sens d'appartenance. La lutte dans laquelle se sont engagés 
les groupes de femmes est l'anti-oppression sous toutes ses formes. Il n'existe pas de formule simple, mais au départ, chaque forme d'oppression doit être reconnue et dénoncée pour que nous puissions la défaire.

\section{Témoignage}

En 1993, fraîchement débarquée dans le milieu, j’ai participé à une rencontre gouvernement-communauté à Toronto, organisée par la Direction générale de la condition féminine de l'Ontario (DGCFO). Près de 200 femmes y étaient. Parmi elles, une dizaine de femmes francophones. Nous avons décidé de nous réunir et de discuter en français des dossiers présentés en vue d'une restructuration des initiatives en matière de violence faite aux femmes. Nous avons par la suite proposé à l'assemblée notre vision, assez simpliste je l'admets, de la communauté divisée en trois groupes: les anglophones, les francophones et les autochtones. Selon notre intervention, la DGCFO devait accorder des argents à ces trois groupes pour les besoins des femmes provenant de leurs diversités.

Quelle réaction de colère notre inexpérience des politiques des groupes de femmes a provoquée! Comment osions-nous mettre dans la catégorie "anglophones» les femmes provenant d'ailleurs (et qui avaient adopté l'anglais comme langue de communication)? Comment osions-nous parler au nom de la communauté francophone s'il n'y avait parmi nous aucune représentante des femmes de minorités visibles? S'il est vrai que notre intervention n'était pas des plus nuancées et notre représentativité limitée, les propos tenus par la suite par des femmes présentes étaient empreints d'un 
fort sentiment anti-francophone. On nous a, entre autres, reproché les politiques du gouvernement $d u$ Québec - la loi 101, etc. - comme si nous étions responsables des décisions de la province voisine. Et plus ça allait, moins on écoutait nos tentatives d'explication, et plus le ton devenait très agressif. Nous avons fini par prendre notre trou, par nous taire. Je suis rentrée penaude, convaincue que j'avais fait la gaffe la plus monumentale de ma vie et que je ne ferais plus cette erreur de dire les besoins et les opinions des francophones. Quand j'ai raconté ça à mes copines d'Ottawa, elles ont rigolé et ont dit: "Maintenant tu sais pourquoi nous ne voulions plus aller à ces rencontres». Ce n'était donc pas la première fois, ce ne fut pas non plus la dernière...

J'ai bien eu le goût de ne plus remettre les pieds à ces rencontres. Cependant, si les femmes d'expression française ne participaient pas à ces rencontres (ou seules celles qui avaient peu d'expérience y étaient envoyées...), de quelle façon allions-nous nous y prendre pour assurer le développement des services en français dans la province, services qui accusaient - et accusent encore - un retard considérable sur les services en anglais? L'agressivité des propos lourds de préjugés démontrait une hostilité certaine envers les francophones: de quoi était-elle faite? Comment pouvionsnous défaire cette hostilité afin de ne pas être condamnées au silence en tant que groupe? Pourquoi aucune participante, ni même les modératrices de la rencontre, ne sont-elles pas intervenues pour dénoncer cet irrespect flagrant, alors qu'il est de mise de soutenir - ou tout au moins de ne pas critiquer - les revendications des groupes minoritaires (femmes de couleur, femmes vivant avec un handicap, lesbiennes, autochtones)? Les femmes francophones n'avaient-elles pas droit à cette considération? Les animatrices étaient-elles d'accord avec ces propos méprisants? Auraient-elles toléré 
que nous tenions des propos dérogatoires envers l'un ou l'autre des autres groupes? Seules deux femmes sont venues, en privé, nous dire qu'elles nous soutenaient.

\section{La population de langue française en Ontario}

"Contrairement à l'idée que l'on se fait souvent, les femmes francophones ne sont pas toutes bilingues, en particulier les récentes immigrantes arrivées ici avec l'espoir de pouvoir vite s'intégrer et travailler,..."
En Ontario, la communauté francophone compte environ 547000 personnes dont la langue maternelle est le français ${ }^{2}$. Il est à remarquer que ce chiffre n'inclut pas les immigrants et les immigrantes dont la langue d'usage est le français et dont le nombre s'élève à 82000 personnes selon les données de l'ACFO, ni les personnes qui ont coché la case «bilingue» lors du recensement, et l'on sait qu'il y a cinq fois plus de francophones qui sont bilingues que d'anglophones ${ }^{3}$. D'après nous, ces statistiques sous-estiment grandement le nombre réel de francophones en Ontario... et nous sommes parfois tentées de nous demander si cette sous-représentation servirait à des fins politiques.

Le premier appel que nous avons reçu au Centre francophone provenait d'un homme au nom français, mais s'exprimant seulement en anglais, qui nous a dit: "Pourquoi des services en français? Il $\gamma$ a seulement dix femmes francophones dans la province et elles parlent toutes anglais!»

Témoignage

Contrairement à l'idée que l'on se fait souvent, les femmes francophones ne sont pas toutes bilingues, en particulier les récentes immigrantes arrivées ici avec l'espoir de pouvoir vite s'intégrer et travailler, étant donné qu'elles possèdent l'une des deux langues officielles du Canada. Cet unilinguisme francophone a souvent des conséquences graves pour les femmes immigrantes qui doivent composer en plus avec le racisme et la xénophobie. 
Quand je suis arrivée au Canada, j'ai rejoint mon mari installé à Toronto. Je croyais qu'avec le français je pourrais me trouver rapidement $d u$ travail, puisque c'est une des langues officielles du Canada. Ma première sortie fut d'aller au dépanneur où je me suis adressée en français au commis. Il s'est mis à m'injurier en anglais, je ne comprenais pas ce qu'il disait. Un autre client a traduit ma demande au commis. J'ai voulu savoir ce que le commis m'avait dit. Le client m'a répondu: "Vous ne voulez pas savoir..."

Témoignage

Le Comité des femmes noires africaines francophones d'Ottawa estime à près de $90 \%$ le taux de femmes africaines d'expression française qui sont sans emploi (COFANF 1997).

S'il y a bon nombre de femmes francophones qui parlent l'anglais, elles sont pour la plupart beaucoup plus habiles dans leur langue maternelle, cela va de soi. Plusieurs femmes impliquées dans les groupes de femmes disent qu'elles participent à peine aux rencontres qui se déroulent en anglais, alors qu'elles sont de véritables moulins à parole lors des rencontres en français.

Si je parle anglais, je me sens moins nuancée, je me sens moins intelligente, je me sens donc moins crédible. Si je parle français, je sens que je dérange et que parfois on ne me comprend pas. Si je demeure silencieuse, je me sens exclue, je sens que je n'apporte rien au groupe... Que dois-je faire?...

Témoignage

\section{Impacts de 1'oppression}

À la suite d'une rencontre d'intervenantes des maisons d'hébergement, ma collègue anglophone me demandait comment il se faisait que les quelques francophones présentes n'avaient pas revendiqué de traduction ou d'atelier dans leur langue. Pourquoi, disait-elle, ne pouvaient-elles pas faire valoir leurs droits? 
"Grandir dans des conditions d'oppression a nécessairement un impact sur sa participation dans le monde. "

"...il y a mille façons de vivre l'oppression intériorisée. Ces choix représentent des mécanismes de survie dans un environnement intolérant..."
Grandir dans des conditions d'oppression a nécessairement un impact sur sa participation dans le monde. En tant que femmes, nous le savons: certaines sont passives, certaines revendiquent, certaines ont peur et souhaiteraient disparaitre plutôt que de dire: «Je suis femme et je veux que vous respectiez mes droits». Et certaines vont travailler à l'encontre des objectifs d'égalité et de reconnaissance des droits des femmes.

Il y a des francophones tellement en «mal-aise» avec leur francophonie qu'elles contribuent à l'oppression et au dénigrement de la francophonie. Elles siègent sur les collectives ou des conseils d'administration et leurs décisions contribuent à limiter l'accès des services aux femmes francophones. Mais à part ces situations extrêmes, il y a mille façons de vivre l'oppression intériorisée. Ces choix représentent des mécanismes de survie dans un environnement intolérant: certaines ont choisi de parler et de vivre en anglais, oubliant même leur langue maternelle; certaines perçoivent les services en anglais comme étant meilleurs, même si elles ne les connaissent pas; certaines n'osent pas afficher leur différence, ne réclament pas de traduction et ne font pas valoir les besoins des femmes francophones, par crainte de soulever la colère des autres (ce qui arrive!), de devoir quitter ou perdre leur emploi (ce qui arrive aussi).

Dans un Centre d'aide et de lutte contre les agressions à caractère sexuel (CALACS), une employée à temps partiel s'occupe de la mise sur pied des services en français. La tâche est difficile. Les femmes de la communauté, peu habituées à avoir accès à des services en français, ne demandent pas de services. $\mathrm{La}$ travailleuse entreprend donc de faire la tournée des écoles, de rencontrer les groupes communautaires, etc. Le travail ne suscite pas une utilisation immédiate de la ligne de crise du centre, en grande partie parce que le tabou sur la violence à caractère sexuel est encore très présent. Elle se sent vite débordée, ses 18 heures/ semaine ne suffisent pas et elle demande plus de temps pour faire son travail. Les deux employées à temps 
partiel embauchées pour faire le même travail dans la communauté anglophone sont aussi débordées et réclament une augmentation de leurs heures de travail. Elles l'obtiennent, mais l'employée francophone ne l'obtient pas. Elle s'est rendue compte, lors des discussions avec la collective, que les membres n'avaient pas même lu les rapports qu'elle avait préparés. Se sentant complètement marginalisée, elle démissionne. (A ce jour, il n'y a pas eu de réembauche pour le travail de développement des services en français dans ce centre. NDLR)

Témoignage

"La tension qui monte dans la salle lorsqu'on parle de francophonie est si vive qu'il faut un échappatoire, une façon d'être ailleurs pendant que l'on aborde ce sujet.»
Certaines sont mal à l'aise et ne peuvent se solidariser avec celles qui réclament, par crainte d'être vues comme radicales ou "séparatistes»; ou elles craignent de perdre leurs acquis ou leur crédibilité, de n'être perçues que comme des porte-parole d'une seule parole : "Ha! She's going to ask for translation again". On entend presque le soupir dans la salle... certaines sont tellement convaincues que ce sera le message qu'elles ne prennent pas la peine de mettre les écouteurs s'il y a traduction simultanée... Ou encore: "Quand j'ai commencé à parler des besoins des femmes francophones, les femmes ont commencé à se lever, à parler entre elles, à fouiller dans leurs sacs. C'est comme si je ne parlais pas...».

Plusieurs d'entre nous avons eu cette même expérience. La tension qui monte dans la salle lorsqu'on parle de francophonie est si vive qu'il faut un échappatoire, une façon d'être ailleurs pendant que l'on aborde ce sujet. Nous avons observé le même phénomène lorsque les femmes autres que celles qui constituent la majorité - comme les femmes vivant avec un handicap ou les femmes immigrantes — soulèvent un sujet qui leur est particulier: les chaises bougent, les participantes parlent entre elles ou se lèvent pour aller chercher un café... Tension ou manque d'intérêt?...

Au-delà de l'impact que l'attitude d'intolérance peut avoir sur les femmes, on peut facilement imaginer cet impact sur le développement des services. Pourquoi les services en français dans les CALACS n'existent-ils que depuis moins de 5 ans dans la plupart des régions? Pourquoi leurs budgets et leurs modes de gestion dans 
plusieurs cas apparaissent-ils comme des recettes infaillibles pour mener à l'échec? Comment se fait-il que moins de 10 maisons d'hébergement (sur 96) disent offrir des services en français?

Pourquoi faut-il rappeler coup après coup au gouvernement ontarien son obligation en vertu de la Loi de 1986 sur les services en français de traduire les documents qu'il rend publics? Même le gouvernement fédéral s'en mêle parfois, organisant des consultations à Toronto, sans traduction, mais offrant aux francophones de les faire transporter à Moncton ou à Montréal pour parler des besoins des femmes francophones... de l'Ontario.

Au niveau communautaire, les femmes francophones rencontrent des obstacles systémiques dans leurs propres regroupements de femmes: l'absence de traduction aux rencontres, de la documentation en anglais seulement, l'obligation pour une ou deux individus de faire valoir les besoins de toute la communauté francophone.

En plus des raisons énumérées plus tôt, les femmes francophones choisissent parfois de se taire parce qu'elles ne veulent pas se retrouver avec toute la tâche à faire.

Comme seule participante francophone au comité organisateur du 6 décembre dans mon coin, je me suis retrouvée avec la même tâche que six femmes anglophones se sont partagée : recruter des porte-parole, des artistes, intervenir dans les médias, préparer du matériel publicitaire, faire circuler l'info...

Elles auraient à faire le travail dans des conditions qui ne leur permettent pas d'accomplir un travail adéquat.

Pendant le processus d'établissement de protocoles d'intervention auprès des femmes victimes d'agressions sexuelles avec la police de $X$, une consultation a été organisée auprès des fermmes de la communauté. Le comité a recruté une femme francophone, à peine quelques jours avant la tenue des consultations, pour faire la publicité et le recrutement des participantes. La lettre d'invitation 
en français qu'on lui a remise était pleine de fautes. Aucune participante francophone ne s'est présentée.

$\mathrm{Ou}$ encore elles ont à faire le travail dans des conditions différentes.

Dans notre région, le comité de coordination régional a accordé des contrats à deux femmes, contrats d'une valeur de 15000 \$, pour former des bénévoles pour mettre sur pied des groupes d'entraide en anglais. Puis, le comité a demandé à une intervenante francophone de faire bénévolement le même travail pour les groupes d'entraide en français.

\section{Le débat sur l'unité nationale et le «Québec bashing»}

La situation s'est empirée au cours des dernières années, à cause du débat sur l'unité nationale, opposant le Québec au reste du pays. À chaque référendum, le phénomène de "Québec-bashing» s'amplifie.

J'étais en train entre Toronto et Ottawa. Derrière moi, un homme francophone et une fermme discutaient en anglais de la question d'unité nationale. Ils ont tenu des propos des plus haineux envers les Québécois et les francophones en général, au point où je trouvais impossible de rester dans mon siège et je suis allée m'asseoir ailleurs. Puis je me suis surprise à penser qu'advenant le pire, mes filles seraient peut-être protégées parce qu'elles portent un nom anglais et qu'elles parlent bien cette langue. J'ai eu honte de mes propres pensées... 


\section{Le groupe «in», le groupe «out»}

«Dans les situations perçues comme conflictuelles, la nuance n'a plus de place."
Dans la situation actuelle de tension sur la question d'unité nationale, les francophones sont tous mis dans le même bateau et les francophones en Ontario sont pris à partie pour les décisions de la province voisine, comme cela s'est passé en '93 et comme cela s'est encore passé récemment dans une rencontre à laquelle participait une collègue. Dans les situations perçues comme conflictuelles, la nuance n'a plus de place. La psychologie sociale nous renseigne sur ce phénomène de groupes d'appartenance, où en situation de compétition, la tendance de voir la composition du groupe opposé comme similaire ou homogène s'intensifie (Worchel et al 1991).

De la même manière, lors de la rencontre de ' 93 , notre petit groupe de femmes francophones a mis toutes les femmes «autres» dans la catégorie "anglophones", à l'exception des femmes autochtones. Il est évident que notre vision de la communauté reflétait que le noeud de l'oppression se situe au niveau de la langue pour nous, alors que pour d'autres le noeud est la couleur de la peau, l'orientation sexuelle ou le handicap. De la même manière qu'il est erroné de notre part de mettre toutes les participantes dans une même catégorie définie seulement à partir de la langue, il est aussi erroné d'ignorer les revendications spécifiques des femmes francophones de l'Ontario à cause des enjeux politiques liés à la province voisine.

\section{Une hypothèse farfelue}

Si je prenais une carte du Canada et que je dessinais une ligne pour séparer la bande inférieure d'environ un quart du pays au sud et trois quarts au nord, et que je décrétais qu'étant donné que la majorité de la population se trouve dans le quart inférieur, dorénavant, il n'y aura de services gouvernementaux — livraison 
"Il n'y a rien pour diviser un groupe (ce qui sert bien le pouvoir en place) comme de souligner que les "privilèges» d'un groupe nuisent au développement des autres groupes.» du courrier, entretien de chemins, etc. - que dans cette partie du Canada, quelles seraient les réactions des Canadiens? Pourraiton demander à ceux et celles qui ne sont pas contents de déménager au sud de cette ligne? Impensable, n'est-ce pas? Il y a des réalités que l'on accepte sans broncher : le coût élevé du service postal, celui du déblaiement de la neige, etc., parce que cela fait partie de la réalité de notre pays. Cependant, en ce qui concerne la francophonie et les coûts qu'on y associe, cette même attitude n'est plus. "Ça coûte cher!" est l'argument qu'on entend chaque fois qu'on ose (si on ose!) demander la traduction simultanée ou tout au moins, la traduction des documents. En ces temps de restrictions budgétaires, on a presque honte de demander la traduction, parce que par notre faute, il y a aura encore plus de restrictions à d'autres services. Il n'y a rien pour diviser un groupe (ce qui sert bien le pouvoir en place) comme de souligner que les "privilèges» d'un groupe nuisent au développement des autres groupes.

Mais s'agit-il de privilèges? Les employées du gouvernement fédéral qui réclament l'équité salariale veulent-elles un privilège ou réclament-elles en vertu de leurs droits à l'égalité en tant que citoyennes? Les garderies accessibles sont-elles un privilège ou ne devraient-elles pas être un droit afin que les femmes puissent aller travailler et sortir de la dépendance financière envers le conjoint ou l'État? La résistance patriarcale s'oppose à l'équité salariale, aux garderies, à l'accès à la justice pour les femmes en invoquant très souvent les facteurs économiques, les coûts exorbitants qu'entraînerait la mise en oeuvre d'une véritable égalité pour les femmes.

De quoi est donc faite cette résistance au développement des services en français, si ce n'est une haine profondément ancrée? Quel sentiment animait la travailleuse qui a dit à ses collègues qui discutaient entre elles en français en se servant un café : «Please! I don't feel like hearing French today"? Aurait-elle dit à une femme qui utilise un fauteuil roulant: "I don't feel like seing a wheelchair today»?... 


\section{Bibliographie}

COMMUNAUTÉ DES FEMMES AFRICAINES NOIRES FRANCOPHONES (COFANF) (1997). Projet: Briser le circuit de la dépendance économique, Ottawa.

WORCHEL, Stephen, Joel COOPER et George A. GOETHALS (1991). Understanding Social Psychology, 5th edition, Brook/Cole Publishing, California.

\section{Notes}

1. Tout au long de ce texte, l'expression «les femmes francophones» ou «les femmes d'expression française», ne s'adresse pas seulement aux femmes nées en Ontario, mais bien à toutes les femmes dont la langue de communication choisie ou d'identité est le français, incluant les femmes immigrantes pour qui le français, sans être la langue maternelle, constitue la langue d'usage au Canada.

2. Office des affaires francophones de l'Ontario, 1998.

3. Selon le recensement de 1996, Statistique Canada, Le Quotidien, mardi le 2 décembre 1997 